\title{
Perception, Exploration, and the Primacy of Touch
}

\section{Matthew Ratcliffe}

\section{Introduction}

Philosophical discussions of perception tend to focus principally on vision. However, there is the concern that what applies to vision may not apply to perception more generally, and that other senses can offer different insights into its nature. ${ }^{1}$ A stronger claim, made by Alva Noë, is that many philosophical treatments of perception not only over-emphasize vision; they are also rooted in a mistaken "photographic model" of vision. Touch, he suggests, offers a better starting point from which to understand the nature of perception:

Think of a blind person tip-tapping his or her way around a cluttered space, perceiving that space by touch, not all at once, but through time, by skillful probing and movement. This is, or at least ought to be, our paradigm of what perceiving is. The world makes itself available to the perceiver through physical movement and interaction. (Noë, 2004, p.1)

According to Noë, all perception is "touch-like", insofar as it involves an active, exploratory process, rather than the passive receipt of information. Perception relies upon "knowledge" of “sensorimotor contingencies". In other words, it incorporates a tacit, non-conceptual appreciation of how various kinds of motor activity affect patterns of sensory stimulation. ${ }^{2}$ For current purposes, I will assume that something along these lines is right: that most or even all perceptual achievements utilize stable relationships between kinds of activity and kinds of sensory change, and that active, tactual perception exemplifies this particularly well. ${ }^{3}$

\footnotetext{
${ }^{1}$ See, for example, O’Callaghan (2007).

2 See also O'Regan and Noë (2001).

${ }^{3}$ I do not go so far as to accept the view that sensorimotor contingencies are sufficient for tactual perception or for any other form of perception. The weaker claim I endorse here is that they are a necessary but not sufficient constituent of most -but perhaps not all- perceptual processes. My discussion does not require endorsement of various stronger claims made by O'Regan and Noë (2001), to the effect that their approach can accommodate all those aspects of perception that others have sought to account for in terms of representations, images, qualia, and so forth (although I confess that I would very much like to dispense with all talk of representations and qualia). In order
} 
On one interpretation of the claim that all perception is touch-like, the different perceptual modalities all shares certain features, but these features are especially salient in the case of touch. So a good approach is to start by reflecting on the structure of touch and then generalize to the other senses. If this is right, then one could just as accurately state that touch is "vision-like", at least when vision is characterized properly. Indeed, J.J. Gibson (1962, p.477) observes that exploratory touch with the fingers is akin to eye movements, and goes so far as to say that "active touch can be termed tactile scanning, by analogy with ocular scanning". On the other hand, even if all sensory perception utilizes sensorimotor contingencies, this does not rule out the possibility that touch remains distinctive in some respect. Perhaps touch is an effective exemplar for enactivism because it depends upon bodily activity to a greater extent than the other senses and/or in a distinctive way. With this in mind, we might consider various claims to the effect that touch is the most fundamental sense, the only sense that is essential to the having of a body, to the capacity to interact with one's surroundings, to animal being (e.g. Jonas, 1954;

O’Shaughnessy, 1989; Fulkerson, 2014).

This chapter addresses the question of whether and how the relationship between touch, the body, and bodily activity might be distinctive. I begin by pointing out a complication for any claim that 'touch is or involves $p$ ': it is unclear what touch is, and any generalizations concerning the nature of touch are susceptible to counter-examples, involving either cases of touch that do not involve $p$ or non-tactual forms of perception that do. Even so, I argue that most uncontroversial instances of touch incorporate bodily activity in a way that is consistent with the sensorimotor contingency view. Following this, I consider a recent defence of the claim that tactual perception utilizes bodily exploration in a distinctive way and to a greater extent than the other externally directed senses. I raise some objections, and suggest that there are insufficient grounds for maintaining that touch is somehow 'more enactive' than other senses. However, this leaves open the possibility that touch relates to the body in a unique way, in virtue of some other characteristic. Hence I go on to address other formulations of the claim that touch is both more fundamental than the other senses and more intimately bound up with our bodily nature. All of

to keep things manageable, I focus on this specific formulation of enactivism. However, many of the points I make are of relevance to other kinds of enactivist approach as well. 
these turn out to be problematic. I conclude that the most plausible case for the primacy of touch involves an appeal to its diversity, rather than to any particular characteristic of touch.

\section{The Nature of Touch}

Generalizations concerning what touch or 'tactual perception' does or does not involve are invariably problematic, as it is unclear what counts as an instance of touch, what does not, and why. Philosophers have proposed various criteria for individuating the senses, most of which further develop one or more of the four criteria proposed by Grice (1966): (i) what is perceived via a sense (e.g. colour, rather than sound); (ii) the distinctive introspectible quality of the sensory experience; (iii) the kinds of stimuli detected; (iv) the composition of sense organs and their connections to the brain. ${ }^{4}$ More recently, Keeley (2002) has distinguished between the tasks of identifying commonsense criteria (which include but are not limited to the relevant phenomenology) and identifying those (non-phenomenological) criteria that are applicable in scientific enquiry. Turning to the latter, he proposes that each sense is receptive to a specific stimulus type (to be construed in terms of the kinds of energy identified by physics) and involves distinctive sense organs. In addition, the organism discriminates stimuli on the basis of a given form of energy without relying on additional means of detection, via mechanisms that are evolutionarily and developmentally dedicated to detecting those specific stimuli. ${ }^{5}$

Perhaps some combination of these or other criteria will succeed in individuating certain senses, but any such inventory is problematic for touch. There is no clearly bounded organ of touch. It utilizes exterior and interior surfaces of the organism, hair, various different types of sensory receptor and, as I will make clear in the next section, a sense of bodily position and movement. Furthermore, touch is not receptive to a specific stimulus type. It is not only a 'pressure sense'. Certain tactual achievements in humans and other organisms depend more specifically upon the detection of vibration, which is not just 'pressure' or 'change in pressure' but a temporally extended, structured pattern of pressure changes (Katz, 1925/1989). It can be added that tactual

\footnotetext{
${ }^{4}$ In what follows, I refer to (i) in terms of the 'content' of tactual experience. I use the term 'content' in a noncommittal way, to mean whatever it is that one perceives through touch - a texture, an object, an occurrence. Although I do not deny that one could perceive something without experiencing it, my discussion of content is concerned more specifically with perceptual experience: I take the content of tactual perception to be whatever it is that we experience through touch. This use of the term 'content' does not imply any commitment to the existence of perceptual 'representations'.

${ }^{5}$ For several other proposals for individuating the senses, see the essays collected in Macpherson ed. (2011).
} 
perception is inseparable from perception of hot and cold, and therefore reliant on at least two distinct forms of energy. Katz (1925/1989, p.165) observes that temperature contributes to recognition of "almost every material", a point that applies equally to texture-perception. For instance, cold surfaces generally feel smoother than warmer ones (Taylor, Lederman and Gibson, 1973, p.260). The view that touch is inextricable from perception of hot/cold is also supported by neurobiological findings. ${ }^{6}$ Some sensory neurons are receptive to heat and to certain mechanical stimuli, as well as chemical irritants (Schepers and Ringkamp, 2010, p.181; Lumpkin and Caterina, 2007). ${ }^{7}$ Of course, one could respond by appealing to cases where hot/cold perception is absent but other aspects of touch remain. However, this does not give us sufficient grounds for maintaining that hot/cold perception is a separate sense, given that selective absences also arise within other established perceptual modalities. For example, loss of colour vision does not amount to a complete loss of vision.

Even if perception of hot/cold were to be excluded from the sense of touch, its reliance on pressure/vibration does not serve to distinguish it from all other senses, as hearing relies on pressure changes in the inner ear. ${ }^{8}$ Of course, one perceives sounds through hearing, rather than pressure changes. The sensory content of audition is thus distinct from the proximal stimulus upon which it depends. But this applies equally to certain instances of touch; what is perceived through touch need not be 'physical contact with something' or 'properties of the entity that one is in contact with'. There are many plausible examples of distance touch. ${ }^{9}$ In using a tool, the most salient content of tactual experience is often not the tool itself but something that is manipulated or investigated with the tool. And many other tactual experiences involve perceiving something through a medium of whatever thickness. When you touch something

\footnotetext{
${ }^{6}$ I say 'hot and cold' rather than 'temperature' because different sensory receptors are responsive to heat and cold. ${ }^{7}$ There is also the question of whether pain should be distinguished from tactual perception. Fulkerson (2014, p.49) argues that pain is distinct from touch, on the basis that only the latter facilitates perception of entities outside of one's own body. For instance, when you have an injection, the pain "is not experienced as a property of the needle". But one could respond by suggesting that, although the pain is not itself experienced as a property of the needle, it does include an awareness of its being externally caused, of one's being hurt by something that is currently in contact with one's body. It also includes some appreciation of the nature of the stimulus, whether it is forcefully or non-forcefully applied, sharp, or blunt. However, although I think it is a mistake to separate hot/cold from touch, I am less convinced in the case of pain. At most, I would be inclined to say that some forms of pain are integral to some tactual experiences, or vice versa.

${ }^{8}$ See Gray (2013) for a recent defence of the view that the sense of touch should be distinguished from perception of heat and cold.

${ }^{9}$ See also Ratcliffe (2012) and Fulkerson (2014) for recent arguments in support of 'distance touch'.
} 
while wearing a glove, you perceive through the glove, rather than perceiving only the material that is in direct contact with your skin. This applies equally to passive touch; one can perceive bumps on the road while sitting on a moving bus. It might be objected that such cases involve perceiving both pressure/vibration and, via that, something else. However, I doubt that this is any more phenomenologically accurate for touch and pressure/vibration than it is for hearing and pressure/vibration or for vision and light. It is uncontroversial that the content of visual perception does not include photons hitting the retina and that the content of hearing does not include pressure changes in the ear. There are no grounds for imposing such a view on those tactual experiences for which it is no more phenomenologically plausible. A further problem for the pressure-sense view is posed by tactual experiences of absence or lack of pressure. For instance, Katz (1825/1989, p.61) offers the example of running one's hand across a brush and perceiving not just the bristles themselves but also the space or "tactual ground" between the bristles. Other examples include exploring a sculpture with one's hands and feeling empty spaces, experiencing a part of one's body as exposed when one's clothes become untucked, and feeling the absence of a wedding ring on one's hand, after taking it off to go swimming. So there is no straightforward relationship between the content of tactual perception and the detection of contact or pressure.

Such examples also render problematic any appeal to a distinctive content and/or an experiential quale or 'what-it-is-likeness' of touch (where the latter requires acceptance of the position that a perceptual experience is not exhausted by its content). The contents of tactual perception are notably diverse. They include objects, textures, things coming into contact with the body, things that are not in contact with one's body, the absence of something, static properties such as shape, and dynamic properties such as patterns of movement. To add to this, it is far from clear what the limits of tactual perceptual content are. When one strokes the hair of one's child as he drifts into sleep, does one perceive a texture, a number of fine strands, hair, a roughly spherical entity with hair attached, a warm, animate body, a person, or a particular person ${ }^{10}$ Some descriptions suggest that the contents of tactual experience can be both extremely diverse and highly detailed. Consider this description that Helen Keller offers of her unusually refined sense of touch:

\footnotetext{
${ }^{10}$ To further complicate things, interpersonal touch is often communicative, and patterns of communicative tactual interaction with another person can feed into the task of perceiving that person through touch. It is unclear whether or how the communicative and perceptual functions of touch are to be separated.
} 
The thousand soft voices of the earth have truly found their way to me - the small rustle in the tufts of grass, the silky swish of leaves, the buzz of insects, the hum of bees in blossoms I have plucked, the flutter of a bird's wings after his bath, and the slender rippling vibration of water running over pebbles. [....] I have endlessly varied, instructive contacts with all the world, with life, with the atmosphere whose radiant activity enfolds us all. The thrilling energy of the all-encasing air is warm and rapturous. Heat-waves and sound-waves play upon my face in infinite variety and combination, until I am able to surmise what must be the myriad sounds that my senseless ears have not heard. (Keller, 1908/2003, pp.40-41)

As for a distinctive experiential quality, it is thoroughly unclear what this might consist of, given the diversity of tactual experience. Perhaps, one might suggest, touch implicates the body in a distinctive way, generating a tactual 'feel' that is not exhausted by perceptual content. But touch involves a wide range of different bodily experiences, involving differing degrees of bodily awareness. Sometimes, one's body or part of it is a conspicuous aspect of the experience but, in other cases, the body is arguably no more conspicuous than it is in visual perception (Ratcliffe, 2012; Fulkerson, 2014). ${ }^{11}$ More generally, touch has all manner of 'feels', regardless of whether or not some of these feels can, in the way that Grice suggests, be distinguished from perceptual content.

For these same reasons, an appeal to sensorimotor contingencies will not suffice to individuate touch. O'Regan and Noë (2001, p.941) maintain that the senses are to be distinguished by the characteristic types of sensorimotor pattern they utilize: "what does differentiate vision from, say, audition or touch, is the structure of the rules governing the sensory changes produced by various motor actions, that is, what we call the sensorimotor contingencies governing visual exploration". However, tactual perception employs the whole body, along with its many and varied capacities for activity. It therefore encompasses a wide range of exploratory strategies,

\footnotetext{
${ }^{11}$ As Katz (1925/1989) puts it, the "object" and "subject" poles of touch vary in salience, while, in the other senses, they are more stable. Martin (1992, p.204) conceives of a tactual experience as a "single state of mind, which can be attended to in different ways". In other words, there is a single, unified experience, with both bodily and non-bodily aspects that can differ in their relative prominence. While I think this is right, it is not specific enough. One might take the line that the content of tactual experience has two different aspects. However, although one's body and parts of it certainly can feature as contents of tactual perception, it would be wrong to restrict the role of the body to perceptual content. The body is also that through which we perceive things tactually; it occupies the role of perceiver (Ratcliffe, 2012).
} 
which can involve any number of sensorimotor patterns. Consider the differences between exploring the location and intensity of a heat source from a distance, discriminating textures (which, for some types of texture, can be achieved either through passive receptivity to something moving across one's skin or through active tactual exploration) and perceiving the shape of an object (which depends more specifically upon active, exploratory touch). Of course, one could say that all of the sensorimotor associations implicated in touch can be distinguished from the "rules" that govern, for instance, visual perception. Then again, they also differ from each other, and so the question arises as why they should be grouped together as a single sense, while those that feature in vision are excluded.

One might respond that tactual discrimination of objects and properties does utilize bodily activity in a sense-specific way. Touch not only involves moving in order to perceive; one also manipulates entities in a number of structured ways, so as to obtain specific types of sensory information. An account along these lines is proposed by Fulkerson (2014), who suggests that what distinguishes a unitary sense from a combination of senses working together is that a sense 'binds' various 'features' into unified perceptual 'objects'. Tactual perception, he says, achieves this in a unique fashion; it is the only sense that involves exploratory binding. I will further discuss Fulkerson's proposal in the next section, in addressing whether some forms of touch are 'more enactive' than other forms of sensory perception. But, for now, I want to note that this approach does not succeed in distinguishing touch from other forms of perception. Fulkerson (2014, p.33) proposes that "haptic perception is unified in virtue of the fact that all of its constituent physiological systems work together to assign sensory features to the same set of objects". However, this clearly does not apply to all uncontroversial cases of touch. Active and passive touch both involve variably sophisticated degrees of discrimination, and do not always facilitate unified perception of an 'object'. So the claim has to be that some cases of touch involve $p$, rather than that all cases of touch involve $p$. The question therefore arises as to why those instances or types of touch that do not involve $p$ still qualify as 'touch'. Their identification as such rests upon an implicit or explicit understanding of what touch is, which is independent of and presupposed by the exploratory feature-binding account.

\section{Touch, Movement, and Exploration}


To some extent, the problem of individuating touch can be circumvented by addressing what a wide range of uncontroversial cases involve, or by making claims about a specific subset of tactual perceptions. For the most part, it is clear that touch cannot be extricated from a sense of bodily position and movement. 'Tactile' perception is sometimes distinguished from 'tactual' or 'haptic' perception, where the former relies only upon sensory receptors in the skin, while the latter also includes proprioception and kinaesthesis. It is clear, I think, that the sense of touch should be construed as 'tactual' rather than just 'tactile'. It is uncontroversial that we rely upon patterns of bodily activity in order to perceive various different properties through touch. Certain textures can be perceived either by moving against a surface or by that surface moving against one's body. Here, only a weak claim applies: some token perceptions of that texture depend upon bodily activity, although a texture of that type can also be perceived in other ways. However, other types of property, such as the shape of a three-dimensional object, are (other than in a few simple cases) only perceivable through exploratory touch. Now, the mere observation that touch depends upon bodily activity is compatible with a model where 'tactile' and 'bodily' information are independently obtained and only subsequently combined so as to facilitate more sophisticated perceptual discriminations. If that were right, it would be debatable whether 'touch' is to be identified with the initial 'tactile' achievement or with the 'haptic'/'tactual' achievement that follows. But it is not right, given that self-initiated movements also shape sensory processes and regulate incoming sensory signals. Consider the example of self-tickling. Most people are unable to tickle themselves, as the perception that arises due to self-stimulation is somehow different, less intense. This difference between self- and other-induced perceptions is not attributable to information about self-produced bodily movement modifying an already given pattern of sensory activity. Rather, the initiation of movement attenuates peripheral sensory signals, and it is not that all incoming sensory signals are affected in the same way; initiation of specific actions modifies "precise sensory signals" (Blakemore, Wolpert and Frith, 2000). The point is not specific to self-touch and applies to touch more generally: sensory processes are regulated in a non-conscious way (and perhaps a conscious way too) by anticipated and actual bodily activities. Indeed, there is evidence to suggest that the same perceptual changes can be brought about either through changes in the pattern of sensory stimulation or through bodily activity; the two variables are "in principle fully interchangeable" (Saig et al., 2012, p.14030). ${ }^{12}$

\footnotetext{
${ }^{12}$ What is perhaps not so clear is when actual and anticipated motor activities lead to sensory attenuation. On
} 
It might be objected that this applies only to active touch. Furthermore, passive touch -which is not reliant upon bodily activity- can similarly facilitate sophisticated and fine-grained perceptions. ${ }^{13}$ Fulkerson (2014) offers the examples of feeling a bug crawl along your arm and feeling your pet cat jump upon and then walk across the bed you are lying in. It is debatable whether such percepts are exclusively tactual in origin though. Try pressing a coin against your palm with your eyes open, and then again with your eyes closed. In the first case, it might seem that you perceive a clearly bounded circular shape through touch, unaided by vision, but the perception is less clearly defined when your eyes are closed. However, regardless of how discriminating passive touch might turn out to be, I do not think it poses a problem for the view that touch is inextricable from a sense of one's body and what it is doing. Both passive and active forms of touch involve proprioception; percepts generally include some sense of bodily location. As for movement, if one lacked all sense of the distinction between self-initiated movement and being acted upon by an external force, it is not that active touch would be lost and passive touch retained. Passive touch involves the sense that a percept is not attributable to selfinitiated movement or, where something unexpected brushes against your hand as you reach for an object, that it is not an anticipated consequence of one's movements. In the absence of any ability to distinguish self-initiated from other kinds of movement, one would be just as unable to experience passive touch. Any remaining experience would be indeterminate, neither active nor passive. The discriminative power of both active and passive touch relies upon a sense of what, if anything, one is doing relative to a perceived entity and whether or not any movements are selfinitiated. In both cases, the ability to perceive would be dramatically curtailed by a severing of the link between sensory stimulation and information concerning the presence or absence of selfproduced movement.

phenomenological grounds, it is arguable that the converse can equally occur. If you stick your hand into a hole, expecting to encounter something slimy and unpleasant, the subsequent tactual encounter with gunge remains a conspicuous one, perhaps more so than if it had not been anticipated it at all. In contrast, if you hold out your hand to receive some coins, the perception of their falling onto your palm is less salient than when something is dropped onto it in the absence of any expectation (an example that straddles the distinction between active and passive touch). Hence conscious anticipation is sometimes associated with attenuation and sometimes not, in a way that is closely related to the affective content of tactual experience. It is also unclear whether attenuation is specific to active touch. It seems plausible to maintain that two instances of passive touch involving the same patterns of sensory stimulation could differ considerably, depending on whether or not something is anticipated and whether or not it is welcome.

${ }^{13}$ Hence it is unhelpful to state, as O'Regan and Noë (2001, p.939) do, that perceiving is a "way of acting"; this obscures the differences between active and passive forms of touch. 
Hence it would seem that the structure of tactual perception is consistent with the sensorimotor contingency view. What one perceives is dependent upon how changing patterns of stimulation relate to bodily activity (or to a lack of bodily activity), rather than the fusion of two prior percepts. The same pattern of sensory stimulation could result in two quite different percepts, depending on whether and how one is moving, and whether a movement is self-initiated. Nevertheless, it is questionable whether this applies to all instances of touch. Take the case of heat perception. When one reaches out with one's hand to explore the proximity and temperature of a nearby heat source, the relevant information is indeed extracted from how patterns of stimulation change in response to bodily movements. What, though, should we say about the simple feeling that 'I am hot' or 'it is hot in this room'? Even if heat perception is ruled out as an instance of touch, many other tactual experiences similarly involve immersion in a diffuse medium. Take the example of swimming under water and, as one does so, exploring the textures of marine plants with one's hands. Here, it seems right to say that one retains some kind of tactual awareness of the water surrounding one's body. But the water is a tactual background in the context of which one has localized tactual experiences of the plants, rather than something one 'encounters' as an object of active or passive touch. And it is not clear that all-over bodily experiences of immersion in a medium can be satisfactorily accounted for in terms of sensorimotor couplings. Such media might be better conceived of as contexts within which sensorimotor couplings operate, media that are - to some extent - compensated for by sensorimotor processes.

We should also consider the possibility that touch is somehow 'more enactive' than other senses. So far as I know, the clearest and most sophisticated statement of that view is Fulkerson's (2014) account of exploratory feature-binding. Fulkerson maintains that the senses bind "features" into "objects", and that this distinguishes them from multi-sensory phenomena, which involve associations between already bound percepts. Drawing on work by Susan Lederman and colleagues, Fulkerson notes that active touch involves a range of "exploratory procedures", each of which is specific to certain kinds of property (see e.g. Klatsky and Lederman, 2002). Some such procedures, he proposes, facilitate "exploratory binding". While the other senses involve feature-binding, they do not rely upon manipulating the environment in the way that touch does. 
Hence at least some tactual achievements are distinctive, as perceptual coherence is achieved by acting upon things in specific, temporally extended, and often complicated ways. We might therefore say that touch is 'the most enactive' of the senses. ${ }^{14}$

My first worry about this proposal is that the nature of 'features' and 'objects' is not made sufficiently clear. Fulkerson does not limit his use of the term 'object' to three-dimensional, temporally enduring entities, and neither is he referring simply to 'objects of perception', meaning 'whatever it is that one perceives'; unbound features and objects can equally be 'objects of perception'. Within the category of 'objects', Fulkerson includes spatiotemporal objects, sounds, and also smells: "In vision, a set of unique features -including color, shape, texture, and motion- are all predicated, or bound, to visual objects. In audition, a range of auditory features are assigned to individual auditory objects, typically thought to be sounds. In olfaction, features or qualities are predicated to odors" (2014, p.35). However, it is not clear to me that the unified 'objects' of hearing are restricted to 'sounds'. As a motorbike roars past, does one simply hear a roaring sound or does one hear the 'sound of something moving past' or, even more specifically, the 'sound of a passing motorbike'? If we at least sometimes hear 'the sound of $p$ ', then it is arguable that sounds are features, of a kind that are sometimes bound to objects by audition and sometimes not. It is similarly unclear why smells should be objects. Suppose I encounter a 'cheesy smell'. It is not that I experience a particular object, which just happens to have cheesiness as one of its contingent properties. If the cheesiness is subtracted from the smell, then I do not experience that smell at all. The smell is inseparable from its cheesiness; it is a 'smell of cheesiness'. This is quite different from perceiving a red cup, where the cup could equally have been blue rather than red. But let us suppose, for the sake of argument, that smells can be decomposed into features. Their status as objects would still be in question. Consider textures, which Fulkerson takes to be features of tactual objects, rather than objects in their own right. As noted by Taylor, Lederman and Gibson (1873, p.252), touch is receptive to properties such as "temperature, hardness, roughness, elasticity, stickiness, slipperiness, rubberiness" and so forth, which are "together perceived as texture". So, if texture is a feature rather than an object, then it

\footnotetext{
${ }^{14}$ Fulkerson's discussion of touch is not principally concerned with enactivism, and he expresses reservations about the general applicability of a sensorimotor contingency approach. However, those reservations principally concern whether the approach can supply an account of perception that allows us to dispense with any appeal to representation or imagery, rather than whether sensorimotor contingencies are at play at all.
} 
is a feature assembled out of other features. If features can have features, then the possibility of decomposing a smell into component features does not suffice to make it an object rather than a feature.

Without a clearer account of features and objects actually are, it is difficult to rule out various other candidates for exploratory feature-binding, which are either not exclusively tactual or not tactual at all. Take the experience of moving a piece of chocolate around in one's mouth, manipulating it with one's tongue, and sampling its taste, texture, and smell. This seems to involve a unified perceptual object, to which taste, texture, and smell are equally bound. Much the same can be said of the relationship between certain smells and what emits them. Suppose I smell something nearby and explore my surroundings to seek out the source. I eventually pick up a rotten apple from the floor and sniff it, while moving it towards and then away from my nose. At some point during this process, I come to experience the smell as a property or 'feature' of the apple that I see, rather than a separate olfactory percept that is associated with a visual percept. Exploratory strategies are similarly implicated in auditory perception. In shaking a ball to discern its contents and repeatedly hearing a ringing sound, one experiences the sound of the ball or of something inside it. Both smelling the apple and hearing the ball involve assigning properties to objects by means of exploratory procedures, and it is unclear that the resulting percepts are any less unified than the objects of touch. One might respond that the two were experienced as separate to begin with and therefore remain separable. However, this applies equally to tactual exploration. Simple, brief movements seldom suffice to unify percepts in the way that Fulkerson describes, and the features that exploratory procedures bind together may start off as separate tactual percepts. Hence, given the way in which Fulkerson distinguishes features from objects, it is unclear that feature-binding in touch differs from various multisensory achievements.

Of course, one could maintain that combinations of other senses only occasionally bind features into objects, but again that applies to touch as well. According to Fulkerson, the unified 'objects' of exploratory touch are three-dimensional objects in the familiar sense of the term (although it is debatable how fine-grained our tactual object-recognition capacities are). However, even in humans, touch is not principally an object-recognition sense. For the most part, it involves the detection of 'features', such as textures. It can be added that tactual object-recognition via touch 
may rely upon other senses as well. Cardini et al. (2010) report that whether someone feels a stimulus touch one or both of her cheeks can be influenced by showing her photographs of a face being touched in one or the other way, an effect that is most pronounced when the face she sees is her own. And Katz (1925/1989, pp.136-7) observes that the more general ability to make finegrained textural discriminations often relies on vision, even where it might seem that differences are detected through touch: "the dissimilarity of the visual impressions can make the tactual impressions appear different to some extent, even when the hand alone notices no differences, and one thus must speak of the strong effects of visual associations". He further suggests that, where visual input is absent, it may remain the case that visual imagery is generated, and that this increases the specificity of experienced content. However, if it is at least accepted that not all tactual object recognition is multisensory, perhaps it is plausible to maintain that touch is distinctive in being the only unitary sense to employ exploratory feature-binding. Then again, in the absence of other criteria to specify why touch indeed is a unitary sense, exploratory featurebinding could just as well be taken to cast doubt upon its unity. And, even if it is granted that touch is a single sense while all other cases of exploratory feature-binding are multisensory, it would remain the case that exploratory feature-binding is not unique to touch (unless, that is, the concepts of 'feature' and 'object' can be refined so as to exclude all the multisensory cases while retaining the tactual ones).

Hence I am sceptical of the possibility that touch involves exploratory activity in a way that is qualitatively different from other forms of sensory perception. Nevertheless, it is plausible to maintain that there is a quantitative difference. Indeed, touch may well encompass more exploratory strategies than the other senses combined, or perhaps a more diverse range of strategies (although, to fully develop this position, one would need to supply criteria for distinguishing and comparing types of exploratory strategy). But, even if this is so, it is wholly symptomatic of the fact that the 'organ' of touch encompasses the motor capacities of the entire body. The other senses rely on more localized structures that occupy fixed bodily locations, thus constraining the kinds of sensorimotor pattern they can take advantage of. As Gibson (1968, p.42) remarks, "the eyeball is 'all of a piece', but it is an unusual sense organ", while the "organ of touch is dispersed over the whole body". It can be added that the hands alone have a degree of 
dexterity and flexibility that facilitates all manner of specialized exploratory procedures. ${ }^{15}$ Even so, none of this implies a qualitatively distinct relationship between perception and bodily activity. It is just that other senses rely on fewer and perhaps more neatly circumscribed sensorimotor patterns than touch.

\section{The Primacy of Touch}

Might there remain something distinctive about touch, something that I have so far failed to acknowledge? Independently of recent enactivist literature, various authors have claimed that touch is more intimately associated with the body than other senses, and uniquely indispensable to our engagement with the world. It is thus said to be our primary, most primordial, or most fundamental sense. Several subtly different primacy claims are to be distinguished, most of which are questionable and/or insufficiently clear. Sometimes, emphasis is placed upon the unique contribution of touch to our appreciation of force, contact, pressure, or causation, something that is essential to our sense of being situated in a world as one body amongst many. For instance, Jonas (1954, p.507, p.516) details the many virtues of sight, but adds that the "objectivity" gained via sight comes at the cost of "causal connection", something that does not feature in visual perceptual content. He adds that touch has a kind of primacy amongst the senses, as it is the only sense "in which the perception of quality is normally blended with the experience of force". As such, it is the sense through which "the original encounter with reality as reality takes place"; "the true test of reality". Of course, if one conceives of vision as an active, exploratory process, the disanalogy no longer holds; seeing does not involve a "selfcontained subject" passively surveying a "self-contained object", in the way Jonas describes. Even so, it seems plausible to suggest that vision does not incorporate force-perception. Then again, vision does encompass certain experiences of acting and at the same time being acted upon, which could equally be construed in terms of an "encounter with reality". In interpersonal contexts, one's gaze upon another is perceived to have a certain effect, and vice versa. We can add that tactual experiences of interaction and reciprocity are not invariably reliant upon physical contact with the relevant object, or upon manipulating it. It is unclear why those forms of touch that do involve 'force' perception should take pride of place over other ways of encountering and

\footnotetext{
${ }^{15}$ See Radman (2013) for several relevant discussions of the hand and its capacities.
} 
interacting with reality, unless it is simply assumed from the outset that force is central to our grasp of the real.

Closely related to the 'force' view is the claim that our concept of causation originates in touch, a view that Jonas also seems to subscribe to. More recently, Wolff and Shepard (2013) have offered a detailed statement and defence of this position, maintaining both that our concept of causation originates in an appreciation of force, and that we obtain our appreciation of force through touch. ${ }^{16}$ It is questionable, however, whether our concept of causation originates exclusively in an experience of force. Plenty of other experiences involve a sense of interaction and reciprocity: I shut my eyes to avoid the light of the sun; I hear a loud noise that hurts my ears; and so forth. And do we not detect causation as we enter a hot room and gradually become uncomfortably hot ourselves? Furthermore, perceptions of force such as being shoved hard by somebody are not exclusively tactual in nature, and neither is it clear that they are primarily tactual. One equally hears a noise, experiences a sudden change in the pattern of visual sensation, and loses one's sense of balance. In the absence of changes in the pattern of visual stimulation that are consistent with being suddenly moved by an outside agency, would one still have the same, unambiguous experience of being acted upon? It is also debatable what our concept of causation actually consists of, whether it is homogeneous, and whether all typical people operate with the same concept. So I am doubtful of the claim that an appreciation of causation depends exclusively or primarily on a subset of tactual experiences that involve force-perception.

Moreover, I question the assumption that tactual experiences, of the kind that do involve physical contact with an object of perception, incorporate the simple perception of 'force' or -as others have suggested- 'pressure' ${ }^{17}$ I think that 'perception of pressure through touch' is an abstraction from a diverse range of tactual experiences that are much richer in structure, most of which do not incorporate an isolable experience of 'pressure'. Many tactual experiences of physical contact are affectively charged. One feels the pleasantness of a caress, rather than first feeling the caress and then having an emotional response to it. This observation is consistent with the

\footnotetext{
${ }^{16}$ Interestingly, Wolff and Shepard (2013) suggest that the evidence supports a patient- rather than agent-oriented account of force perception. Hence, on their view, the primacy of touch relates to our being acted upon by our surroundings, more so than our acting upon them.

17 The terms 'force' and 'pressure' do not mean the same thing. However, for current purposes they are interchangeable, as the concerns I raise apply to both.
} 
finding that certain parts of the body have sensory receptors that are specifically dedicated to pleasant touch (e.g. Olausson et al. 2010; Farmer and Tsakiris, 2013). However, tactual experiences are not simply more or less pleasant than each other. Consider the experience of squeezing a large, warm piece of rubber, an experience that might well be pleasant in some way. Now suppose that the patterns of pressure and tactual stimulation are identical to those involved in squeezing one's naked spouse. The two perceptual experiences would still be quite different in character, and in a way that is not simply attributable to one's being more 'pleasant' than the other. A range of different affective tendencies and patterns of anticipation serve to shape the relevant experiences, and the fact that both involve physical pressure gives us no grounds for maintaining that both actually include the same 'experience of pressure'. The fact that a perceptual experience depends upon an object of perception exerting physical pressure on one's body does not imply that the content of the experience includes pressure per se. I grant that certain perceptual contents may approximate a decontextualized, affectless perception of pressure more closely than others. But on what grounds should we regard these as more fundamental to our grasp of reality than all those other tactual experiences that together encompass various different significant relationships and interactions with the surrounding world? As noted earlier, it is also arguable that touch is a vibration sense, rather than just a pressure sense. This further distances us from the view that the kind of reciprocity with the environment that it facilitates is most centrally a matter of feeling and exerting pressure.

Other primacy claims place more emphasis on the inextricability of touch from the having of a human or animal body. For instance, O’Shaughnessy (1989, pp.37-8) states that touch is "essential to the animal condition as such, something which seems to follow from the fact that tactile-sense is broad enough to overlap with the sheer capacity for physical action on the part of its owner.....in touch we directly appeal to the tactile properties of our own bodies in investigating the self-same tactile properties of other bodies". ${ }^{18}$ However, the intuitive appeal of such claims rests upon a failure to distinguish three things: physical contact; a sense that utilizes physical contact; and a sense of physical contact. It is trivially true that being a part of the physical world involves coming into physical contact with other things. But it does not follow

\footnotetext{
18 The view that touch is essential to animality can be traced back to Aristotle. See Freeland (1985) for a good discussion.
} 
from this that the perceptual capacities of an animal body must be sensitive to physical contact. Martin (1993, p.211) acknowledges the possibility of a 'hypothetical jellyfish', which has bodily sensations but does not distinguish its own body from its surroundings. We can add that such a creature need not have sensory receptors on or near its external surfaces. Even so, it could still have an internally located perceptual system of sorts, one that responded to different kinds of stimuli with different motor activities. Candidate stimuli include magnetic fields, acidity, temperature, and chemical signatures that indicate the presence or absence of nutrition sources. So having a body and interacting with one's environment do not require perception of physical contact or even a sense that utilizes physical content with organismic boundaries. One might respond that hypothetical jellyfish perception of this kind still counts a form of touch. However, if one were to accept that, one's inclusion criteria would have to be so permissive as to include every other kind of actual and conceivable sensory achievement. 'Touch' would become synonymous with 'any way of sensing any property of one's environment'. So the animality claim is either false or it is trivially true and uninformative.

Even if we restrict ourselves to the case of human touch, there are insufficient grounds for thinking that touch has a kind of priority, in virtue of its facilitating a sense of confrontation between one's body and another body. Again, it is important to stress the distinction between relying on physical contact in order to sense something and having a sense of physical contact. (By analogy, vision is reliant on photons coming into contact with the retina, but does not include a sense of something coming into contact with the retina.) In fact, many tactual experiences do not involve a clear sense of something as distinct from one's body. Ihde (1983, pp.96-7) offers the example of sitting on a couch and having a diffuse, non-localized, all-over feeling of comfort, which involves a blurring of the boundaries between one's body and its proximal environment. More generally, touch contributes to the sense of being immersed in a medium of one or another kind, something that need not involve clear differentiation between one's body and its surroundings. This, Ihde suggests, is illustrated by the contrast with those experiences that do involve a pronounced sense of the distinction between one's body and its surroundings. Immediately after one has jumped into a cold lake, one experiences a contrast 
between oneself and a surrounding medium that is more usually lacking. ${ }^{19}$ Another example, discussed by Gibson (1962, p.480), is the "feeling of cutaneous contact with the earth", as we stand and walk. This is clearly a tactual experience of sorts, but one that is distinct from both active and passive touch; it is "a means of registering the stable environment with reference to which both the movements of one's body and the motions of objects occur". Hence some forms of touch constitute or at least contribute to a wider phenomenological context, within which more localized passive and active contact experiences arise (Ratcliffe, 2013). Why should a subset of these localized experiences, rather than a variable sense of being 'immersed' or 'grounded', be most fundamental to our grasp of body, world, and/or the relationship between them? So far as I can see, no argument has been offered. Furthermore, it would be problematic to maintain that touch is primordial because it constitutes a variably differentiated perceptual ground and, at the same time, because it facilitates localized experiences of contact and difference. These are quite different kinds of perceptual achievement, and the acceptance of both as fundamental would suggest that the 'perceptual encounter between body and world' is multifaceted, rather than grounded in some singular accomplishment that is unique to a form of touch. Once that much is conceded, a diverse range of other tactual and non-tactual perceptual achievements could just as well be added to the list. ${ }^{20}$

The diversity of touch also poses problems for the view that touch is somehow constitutive of bodily experience in a way that other senses are not. Katz (1925/1989, pp.126-7) considers the experience of self-touching and remarks that "the mutual perception of two body parts having the same sensory organs is unique; there is nothing like it outside of the skin sense....the moving part of the body feels the motionless part as object". He also addresses how the "subject" and “object" poles can reverse during self-touch; as one explores one's own body tactually, different parts of the body alternate between the roles of perceiver and perceived. In a similar vein,

\footnotetext{
${ }^{19}$ In this example, the experience of contrast is largely attributable to perception of cold. Hence one might seek to further support the view that touch involves undifferentiating immersion in a medium by distinguishing tactual perception from perception of hot/cold. I have argued that hot/cold perception is more plausibly regarded as integral to touch. Even so, this remains compatible with the view that tactual perception only occasionally involves a salient contrast between one's body as a whole and one's surroundings.

${ }^{20}$ One might further speculate that the sense of coming into contact with something distinct from oneself and of the boundary between the two is partly attributable to vision. If tactual experiences involve varying degrees of differentiation and -as suggested earlier- visual input shapes tactual perception, it could be that, in some of those cases where there is a sharp distinction between perceiver and perceived, that distinction is partly attributable to seeing two clearly distinct, clearly bounded entities come into contact with each other.
} 
Husserl (1952/1989, Section Two, Chapter 3) maintains that touch (and only touch) is indispensable to the experience of having a body; the body (Leib) is "constituted originarily only in tactuality" (p.158) ${ }^{21}{ }^{21}$ However, the question again arises as to which tactual achievements are fundamental to human bodily experience and which are not. Rather than appealing to the case of one hand touching the other, where the two hands are experienced as distinct and as occupying reversible perceptual roles, we could just as well prioritize experiences such as rubbing one's palms together and experiencing a unified tactual percept rather than separate, albeit interdependent, perceptions of each hand (Ratcliffe, 2013). So my general worry about all these proposals is that they take the form 'a certain kind of touch, $p$, is primary, in virtue of $x$ ', when one could just as well say 'a certain form of touch $q$, is primary in virtue of $y$ '. No independent grounds are supplied for construing our bodily experience or our relationship with the world as principally a matter of $x$ and not of $y$. It is either asserted or assumed, often amounting to nothing more than a hazy intuition. Furthermore, in some cases, it is not clear whether $x$ is even integral to the relevant tactual achievements, as in claims concerning force or pressure.

Hence the sheer diversity of touch renders general priority claims on behalf of touch either unclear or implausible, and there are also insufficient grounds for giving pride of place to any one subset of tactual achievements. Nevertheless, I think this adds up to a compelling reason for accepting a different formulation of the view that touch is more fundamental to human life (and, indeed, to most animal life) than the other senses. In short, touch accommodates so much that if every one of those sensory achievements encompassed by it were absent, a human life would be unsustainable. ${ }^{22}$ This does not apply to the other established senses. Taylor, Lederman and Gibson (1973, p.270) suggest that:

The 'reality' of the touch sensation may possibly be related to the multimodal nature of touch. A thing seen and heard is more 'real' than is the disembodied voice of a singer heard on a stereo system. A thing touched may be at once sensed as a vibrating object, a warm one, and a hard

\footnotetext{
${ }^{21}$ Merleau-Ponty's various discussions of the perceptual reversibility between subject and object, and of its profound phenomenological implications, are indebted to both Katz and Husserl (e.g. Merleau-Ponty, 1945/1962; 1968).

${ }^{22}$ This is not to deny that people can and sometimes do suffer very substantial impairments of the sense of touch. See, for example, Cole $(1991 ; 2004)$ for interesting discussions. However, I know of no recorded case where a living person who is able to communicate has suffered complete loss of all aspects of the sense of touch throughout the entire body.
} 
one.......By exploring freely, one obtains a succession of independent chunks of information about the object, such as could only in very unlikely circumstances have been produced by anything other than a real object.

I think this is the right approach to take. If touch is the 'test of reality', it is because touch encompasses so many different ways of encountering an entity, which relate to each other and to the deliverances of the other senses in a cohesive way. Similarly, if touch is central to our appreciation of force, pressure, or causation, it is because touch provides us with a wide range of perceptual encounters to abstract from, rather than a singular experience of collision. And, if touch is inextricable from bodily experience to an extent that the other senses are not, it is because touch includes many different ways of experiencing one's body, in relation to itself, to the surrounding world, and to other people. The ubiquity and indispensability of touch becomes even more apparent once it is acknowledged that what we perceive through one sense includes an appreciation of what could be perceived by means of other senses. A visually perceived cup looks graspable; a surface looks smooth to the touch (Husserl, 1952/1989; Merleau-Ponty, 1945/1962; Noë, 2004; Ratcliffe, 2008, 2015). Tactual possibilities permeate all experience. For instance, they are integral to the visual appreciation of something as here, now, in close proximity to oneself. If a visually perceived entity did not offer various kinds of tactual possibility, it would appear strangely distant, not quite there, somehow lacking (Ratcliffe, 2013). Furthermore, touch not only gives us multiple actual and potential entry points onto a given object. It offers multiple ways of engaging with a wider, cohesive situation, in the context of which more localized perceptions arise. It is not that one nudges an object and -through some localized 'reality quale', feeling of pressure, force, or solidity, or anything else that might be claimed to characterize the feel or content of tactual perception- mysteriously comes to grasp the reality of things. Such encounters arise within a wider intersensory situation, involving both a variably differentiated sense of immersion in one's environment and a coherent framework of actual and anticipated sensory experiences, many of which are 'tactual' in nature.

So, in a way, touch is the 'test of reality', but only because it encompasses so much and therefore has an unfair advantage over the other senses. It is not the case that some form of touch involves a singularly intimate relationship with the world, perhaps in virtue of its being 'embodied' or 
'enactive' in a distinctive manner. Given the heterogeneity of touch, the question arises as to whether it should be regarded as a single sense at all, and Taylor, Lederman and Gibson (1973) attribute its diversity to its multimodality. One option is to adopt a 'waste-basket' model of touch: touch includes 'everything that is left over', once we have classified those sensory achievements that are notably circumscribed with respect to sense organs, stimuli, contents, and associated bodily activities. But I think that would be premature. Even if no criteria suffice to isolate all instances of tactual perception and only instances of tactual perception, it is plausible to maintain that what we call 'touch' has a degree of unity comparable to the other established senses (Ratcliffe, 2012). So any attempt to dismember touch might turn out to be just as applicable to them (Fulkerson, 2014). Perhaps the most pressing issue to address concerns the nature and extent of multimodality. It could be that intersensory commerce and interdependence are so extensive that the senses should not be 'individuated' at all. By analogy, 'touch' itself encompasses various different kinds of sensory achievement, which are not usually categorized as separate senses. Given this, I will phrase my conclusion in a way that allows for an agnostic stance towards the 'sense of touch': what we currently refer to as touch has a kind of primacy over the other senses, in virtue of its accommodating so much, but it would be a mistake to further insist that touch has this primacy in virtue of any singular characteristic.

Acknowledgements: Thanks to Matthew Fulkerson and an anonymous referee for commenting on an earlier version of this chapter.

\section{References}

Blakemore, S.J., Wolpert, D. and Frith, C. 2000. Why Can't You Tickle Yourself? Neuroreport 11/11: R11-16.

Cardini, F., Constantini, M., Galati, G., Romani, G.L., Làdavas, E., and Serino, A. 2010. Viewing One's Own Face Being Touched Modulates Tactile Perception: an fMRI Study. Journal of Cognitive Neuroscience 23: 503-513.

Cole, J. 1991. Pride and the Daily Marathon. London: Gerald Duckworth.

Cole, J. 2004. Still Lives: Narratives of Spinal Cord Injury. Cambridge MA: MIT Press.

Farmer, H. and Tsakiris, M. 2013. Touching Hands: A Neurocognitive Review of Intersubjective Touch. In Radman, Z. ed. The Hand, an Organ of the Mind. Cambridge MA: MIT Press: 103-130. 
Fulkerson, M. 2014. The First Sense: A Philosophical Study of Human Touch. Cambridge MA: MIT Press.

Freeland, C. 1995. Aristotle on the Sense of Touch. In Nussbaum, M.C. and Rorty, A.O. eds. Essays on Aristotle's De Anima. Oxford: Oxford University Press: 227-248.

Gibson, J.J. 1962. Observations on Active Touch. Psychological Review 69: 477-491.

Gibson, J.J. 1968. The Senses Considered as Perceptual Systems. London: George Allen \& Unwin Ltd. Gray, R. 2013. What Do Our Experiences of Heat and Cold Represent? Philosophical Studies 166: S131S151.

Grice, H.P. 1966. Some Remarks about the Senses. In Butler, R.J. ed. Analytic Philosophy: First Series. Oxford: Blackwell: 133-153.

Husserl, E. 1952/1989. Ideas Pertaining to a Pure Phenomenology and to a Phenomenological Philosophy: Second Book (Trans. Rojcewicz, R. and Schuwer, A.). Dordrecht: Kluwer.

Ihde, D. 1983. Sense and Significance. Atlantic Highlands, N.J.: Humanities Press.

Jonas, H. 1954. The Nobility of Sight. Philosophy and Phenomenological Research 14: 507-519.

Katz, D. 1925/1989. The World of Touch. Trans. Krueger, L.E. London: Lawrence Erlbaum Associates. Keeley, B.L. 2002. Making Sense of the Senses: Individuating Modalities in Other Animals. The Journal of Philosophy 99: 5-28.

Keller, H. 1908/2003. The World I Live in. New York: New York Review of Books.

Kladsky, R.L. and Lederman, S.J. 2002. Haptic Perception. In Nadel, L. ed. Encyclopedia of Cognitive Science. Basingstoke: Palgrave Macmillan: 508-12.

Lumpkin, E.A. and Caterina, M.J. 2007. Mechanisms of Sensory Transduction in the Skin. Nature 445: 858-865.

Macpherson, F. ed. 2011. The Senses: Classic and Contemporary Philosophical Perspectives. Oxford:

Oxford University Press.

Martin, M. 1992. Sight and Touch. In Crane, T. ed. The Contents of Experience. Cambridge: Cambridge University Press: 196-215.

Martin, M. 1993. Sense Modalities and Spatial Properties. In Eilan, N., McCarthy, R. and Brewer, B. eds Spatial Representation: Problems in Philosophy and Psychology. Oxford: Blackwell: 206-18.

Merleau-Ponty, M. 1945/1962. Phenomenology of Perception (Trans. Smith, C.). London: Routledge.

Merleau-Ponty, M. 1968. The Visible and the Invisible. Trans. Lingis, A. Evanston: Northwestern

University Press.

Noë, A, 2004. Action in Perception. Cambridge MA: MIT Press.

O’Callaghan, C. 2007. Sounds. Oxford: Oxford University Press. 
O’Regan, J.K. and Noë, A. 2001. A Sensorimotor Account of Vision and Visual Consciousness.

Behavioral and Brain Sciences 24: 939-1031.

O'Shaughnessy, B. 1989. The Sense of Touch. Australasian Journal of Philosophy 67: 37-58.

Olausson, H., Wessberg, J., Morrison, I., McGlone, F., and Vallbo, A. 2010. The Neurophysiology of Unmyelinated Tactile Afferents, Neuroscience and Biobehavioral Reviews 34/2: 185-191.

Radman, Z. ed. 2013. The Hand, an Organ of the Mind. Cambridge MA: MIT Press

Ratcliffe, M. 2008. Feelings of Being: Phenomenology, Psychiatry, and the Sense of Reality. Oxford:

Oxford University Press.

Ratcliffe, M. 2012. What is Touch? Australasian Journal of Philosophy 90: 413-432.

Ratcliffe, M. 2013. Touch and the Sense of Reality. In Radman, Z. ed. The Hand, an Organ of the Mind. Cambridge MA: MIT Press: 131-157.

Ratcliffe, M. 2015. Experiences of Depression: a Study in Phenomenology. Oxford: Oxford University Press.

Saig, A, Gordon, G., Assa, E., Arieli, A. and Ahissar, E. 2012. Motor-Sensory Confluence in Tactile Perception. The Journal of Neuroscience 32: 14022-14032.

Schepers, R.J. and Ringkamp, M. 2010. Thermoreceptors and Thermosensitive Afferents. Neuroscience and Biobehavioral Reviews 34: 177-184.

Taylor, M.M., Lederman, S.J. and Gibson, R.H. 1973. Tactual Perception of Texture. In In Carterette, E.C and Friedman, M.P. eds. Handbook of Perception, Volume III: The Biology of Perceptual Systems. New York: Academic Press: 251-273.

Wolff, P. and Shepard, J. 2013. Causation, Touch, and the Perception of Force. In Ross, B.H. ed. The Psychology of Learning and Motivation 58. London: Academic Press: 167-202. 